\title{
Bioanalysis
}

\section{Simultaneous determination of trimethoprim and sulfamethoxazole in dried plasma and urine spots}

\begin{abstract}
Background: Trimethoprim-sulfamethoxazole (TMP-SMX) is an antimicrobial drug combination commonly prescribed in children and adults. The study objectives were to validate and apply an HPLC-MS/MS method to quantify TMP-SMX in dried plasma spots (DPS) and dried urine spots (DUS), and perform a comparability analysis with liquid matrices. Results: For TMP the validated range was $100-50,000 \mathrm{ng} / \mathrm{ml}$ for DPS and $500-250,000 \mathrm{ng} / \mathrm{ml}$ for DUS; for SMX, the validated range was $1000-500,000 \mathrm{ng} / \mathrm{ml}$ for both DPS and DUS. Good agreement was noted between DPS/DUS and liquid plasma and urine samples for TMP, while only modest agreement was observed for SMX in both matrices. Conclusion: A precise, accurate and reproducible method was developed to quantify TMP-SMX in DPS and DUS samples.
\end{abstract}

Trimethoprim-sulfamethoxazole (TMP-SMX) is a combination of two antimicrobial agents that inhibit distinct proteins in the tetrahydrofolate synthesis pathway; TMP inhibits the enzyme dihydrofolate reductase and disrupts production of tetrahydrofolic acid, whereas SMX mimics para-aminobenzoic acid and prevents its conversion to dihydrofolic acid via dihydropteroate synthetase [1]. Inhibition of these proteins affects DNA bacterial synthesis and ultimately bacterial growth. When administered together, this drug combination has potent activity against aerobic gram-positive and gram-negative bacteria. In children and adults, TMP-SMX is prescribed to treat urinary, respiratory or GI tract infections [1]. Also, it is commonly prescribed to treat skin and skin structure infections caused by methicillin-resistant Staphylococcus aureus [2] .

Simultaneous quantification of TMPSMX in biological fluids has been performed using HPLC [3-7], HPLC-MS/MS [7,8] and capillary zone electrophoresis [9]. Often these methods have been applied to simultaneously quantify TMP-SMX in human plasma samples collected in adult PK studies. In pediatric PK studies, because of practical limitations regarding the number and volume of blood samples that can be collected ethically, dried blood spot (DBS) sampling and multidrug assays have been proposed as novel tools to improve pediatric clinical trial designs [10]. The advantages of DBS sampling include significantly reduced blood volumes $(10-25 \mu \mathrm{l})$, reduced biohazard risk, ease of storage (room temperature) and improved drug stability [11]. Measurement of drug concentrations in dried plasma spots (DPS) [12-14] and, less commonly, in dried urine spots (DUS) also have been reported [15]. However, measamples has not been reported previously, and measurement of drug concentrations in dried matrix samples in children often has focused on using DBS. Drug measurement in DPS samples has the additional advantage of avoiding the effect of varying hematocrit on sample homogeneity observed with DBS [16] and allows for easy reporting of results as the PK literature frequently focuses on plasma concentrations [12].

The objective of the analyses described herein was to develop and validate an HPLC-MS/MS method for the simultaneous quantification of TMP-SMX in DPS and DUS samples collected in an opportunistic surement of TMP-SMX in DPS and DUS
Daniel Gonzalez, Chiara Melloni, Brenda B Poindexter, Ram Yogev, Andrew M Atz, Janice E Sullivan, Susan R Mendley, Paula Delmore, Amy Delinsky, Kanecia Zimmerman, Andrew Lewandowski, Barrie Harper, Kenneth C Lewis, Daniel K Benjamin Jr, Michael CohenWolkowiez ; on behalf of the Best Pharmaceuticals for Children Act - Pediatric Trials Network Administrative Core Committee ${ }^{\ddagger}$

*Author for correspondence:

Tel.: +1919668 8812

michael.cohenwolkowiez@duke.edu For full affiliations list, please see page 1149.

*See Acknowledgments on page 1147 for listing of committee members. 


\section{Key terms}

Trimethoprim: Bacteriostatic antimicrobial agent belonging to the dihydrofolate reductase inhibitor drug class.

Sulfamethoxazole: Bacteriostatic sulfonamide antimicrobial agent.

Dried plasma spots: Sampling method whereby plasma is spotted on a collection card.

Dried urine spots: Sampling method whereby urine is spotted on a collection card.

pediatric PK study. Clinical samples were then analyzed and a comparability analysis of the dried and liquid matrix samples was performed.

\section{Experimental}

Materials

Free base forms of the study compounds TMP (CAS No. 738-70-5, Batch SZB9352XV) and SMX (CAS No. 723-46-6, Batch SZBC124XV) were purchased from Sigma-Aldrich Corporation (MO, USA) (Figure 1). Stable isotope-labeled forms of the study drugs were used as IS (CDN Isotopes, Inc., Pointe-Claire, Quebec, Canada): $\left[{ }^{2} \mathrm{H}_{3}\right]$-TMP (Lot: E395P36) and $\left[{ }^{2} \mathrm{H}_{4}\right]-S M X$ (Lot: M237P19). Control K2 EDTA human plasma (BioChemed Services, VA, USA) and urine (collected from human volunteers) was centrifuged for approximately $5 \mathrm{~min}$ at $4000 \mathrm{rpm}$ prior to use. Whatman ${ }^{\circledR}$ FTA $^{\circledR}$ DMPK-C were used for the DPS analysis and Whatman ${ }^{\circledR}$ FTA $^{\circledR}$ DMPKC IND dried matrix spotting cards were used for DUS analysis (Whatman Ltd Co., Middlesex, UK; GE Healthcare, NJ, USA Catalog No. WB120224).

\section{Standard solutions}

For the calibration standards, eight concentration levels were prepared for both TMP and SMX in human plasma and urine: $100-50,000 \mathrm{ng} / \mathrm{ml}$ in human plasma for TMP; $500-250,000 \mathrm{ng} / \mathrm{ml}$ in human urine for TMP and $1000-500,000 \mathrm{ng} / \mathrm{ml}$ in human plasma and urine for SMX. The following nominal concentrations were prepared for QC samples for TMP/ SMX in human plasma: 100/1000 (LLOQ and carryover assessments only), 300/3000, 4000/40,000, $40,000 / 400,000$ and 100,000/1,000,000 (dilution linearity assessment only) $\mathrm{ng} / \mathrm{ml}$. In human urine, the following TMP/SMX concentrations were selected for QC samples: 500/1000 (LLOQ and carryover assessments only), 1500/3000, 20,000/40,000, 200,000/400,000 and 500,000/1,000,000 (dilution linearity assessment only) $\mathrm{ng} / \mathrm{ml}$.

For DPS analysis, stock solutions were prepared by accurately weighing the appropriate amount of TMP/ SMX to dissolve in 1:1 (v/v) methanol: dimethyl sulfoxide to obtain $4 \mathrm{mg} / \mathrm{ml}$ and $40 \mathrm{mg} / \mathrm{ml}$ stock solutions of TMP and SMX, respectively. A combined stock solution was then prepared by combining equal volumes of each, resulting in a $2 \mathrm{mg} / \mathrm{ml} \mathrm{TMP}$ and 20 $\mathrm{mg} / \mathrm{ml}$ SMX solution. For DUS analysis, a similar procedure was followed, and a combined stock solution containing $10 \mathrm{mg} / \mathrm{ml}$ TMP and $20 \mathrm{mg} / \mathrm{ml} \mathrm{SMX} \mathrm{was}$ obtained. The stock solutions were stored at $-70^{\circ} \mathrm{C}$ or below. Calibration standards and QC samples were made from these stock solutions.

Calibration standards and QC samples were prepared using human plasma or human urine that was thoroughly mixed, spotted on dried matrix spotting

\section{Trimethoprim}<smiles>COc1cc(Cc2cnc(N)nc2N)cc(OC)c1OC</smiles>

Precursor lon: 291 Product lon: 230

Sulfamethoxazole

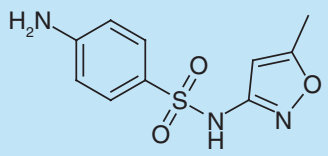

Precursor lon: 254 Product lon: 156 $\left[{ }^{2} \mathrm{H}_{3}\right]$-Trimethoprim<smiles>COc1cc(Cc2cnc(N)nc2N)cc(OC)c1OC(=O)O</smiles>

Precursor lon: 294 Product lon: 123

$\left[{ }^{2} \mathrm{H}_{4}\right]$ - Sulfamethoxazole<smiles>Cc1cc(NS(=O)(=O)c2ccc(N)cc2)no1</smiles>

Precursor lon: 258 Product lon: 160

Figure 1. Study drugs and IS. 
cards and then dried. Calibration standards were extracted fresh daily. A $10 \mu$ l volume per spot was used, except for QCs that were prepared to test the effects of varying spot volume $(5$ and $15 \mu \mathrm{l})$. Calibrated mechanical pipets were used for all volumetric measurements. Calibration curves and QC samples were dried overnight on the bench top and then in a Minigrip zippered bag with desiccant for $24 \mathrm{~h}$ prior to use.

\section{Extraction method}

Initially, $3 \mathrm{~mm}$ punches were used for the dried matrix sample analysis. However, analysis of $3 \mathrm{~mm}$ punches from the center and edge of punches indicated that the TMP and SMX concentrations on the spot were not homogeneous. Therefore, a $6 \mathrm{~mm}$ punch was used to sample the majority of the spot and minimize nonhomogeneity issues. Methanol has been widely used as an extraction solvent for dried matrix sample analysis due to its ability to provide a relatively clean extract by both binding biological matrix to the filter paper on the dried matrix spot card and solubilizing the analyte. Extraction volumes of 100, 200 and $400 \mu \mathrm{l}$ were tested for extraction volumes. The $400 \mu \mathrm{l}$ volume provided the best extraction efficiency. The final extraction method involved punching a $6 \mathrm{~mm}$ spot into a microcentrifuge vial, adding $400 \mu \mathrm{l}$ of IS in methanol, and vortexing for $5 \mathrm{~min}$. Samples were then centrifuged at 13,000 rpm for $5 \mathrm{~min}$. An aliquot $(50 \mu \mathrm{l})$ of each sample was added to a 96-well plate containing $50 \mu \mathrm{l}$ of deionized water.

\section{Liquid plasma \& urine method sample preparation}

An aliquot of sample $(10 \mu \mathrm{l})$ was added to a sample container. IS in methanol $(70 \mu \mathrm{l})$ was added to each sample. Samples were then vortexed for $5 \mathrm{~min}$ and centrifuged at $4000 \mathrm{rpm}$ for $10 \mathrm{~min}$. Sample $(25 \mu \mathrm{l})$ was then added to a 96-well plate containing $75 \mu \mathrm{l}$ of deionized water and analyzed using a C8 HPLC column and MS/MS.

\section{HPLC-MS/MS}

A detailed description of the equipment and settings is provided in Table 1. The Agilent 1200 series HPLC system and Agilent 1290 autosampler were used (Agilent Technologies, Inc., CA, USA). The ACE PFP, $2.1 \times 50$ $\mathrm{mm}, 3 \mu \mathrm{m}$ (Advanced Chromatography Technologies Ltd, Aberdeen, Scotland) analytical column was used. The column temperature was $30^{\circ} \mathrm{C}$. The injection volume, flow rate and run time were $10 \mu \mathrm{l}, 0.75 \mathrm{ml} / \mathrm{min}$ and $3.5 \mathrm{~min}$, respectively. A gradient mobile phase was used: water containing $0.1 \%(\mathrm{v} / \mathrm{v})$ formic acid (mobile phase $\mathrm{A}$ ) and acetonitrile containing $0.1 \%(\mathrm{v} / \mathrm{v})$ formic acid (mobile phase B). During the first $3 \mathrm{~min}$, the percentage mobile phase $B$ increased from 5 to $45 \%$; from 3.01-3.4 min, it was $100 \% \mathrm{~B}$; and for the remainder of the run time (3.41-3.5 min), it was 5\% B. The HPLC system was coupled with an Agilent 6460 series Triple Quadrupole system (Agilent Technologies, Inc., CA, USA). The Agilent Mass Hunter software was used for data acquisition and quantitative analysis. A positive mode electrospray ionization interface was used. The following system settings were used: $350^{\circ} \mathrm{C}$, gas temperature; $10 \mathrm{l} / \mathrm{min}$, gas flow; $50 \mathrm{psi}$, nebulizer pressure; $10.1 \mathrm{l} / \mathrm{min}$, sheath gas flow, $4000 \mathrm{~V}$ capillary voltage; 140/160 V TMP/SMX, fragmentor values and 25/15 V TMP/SMX, collision energy values.

Liquid plasma and urine concentrations were measured using validated HPLC-MS/MS methods.

\section{Table 1. Description of equipment and settings.}

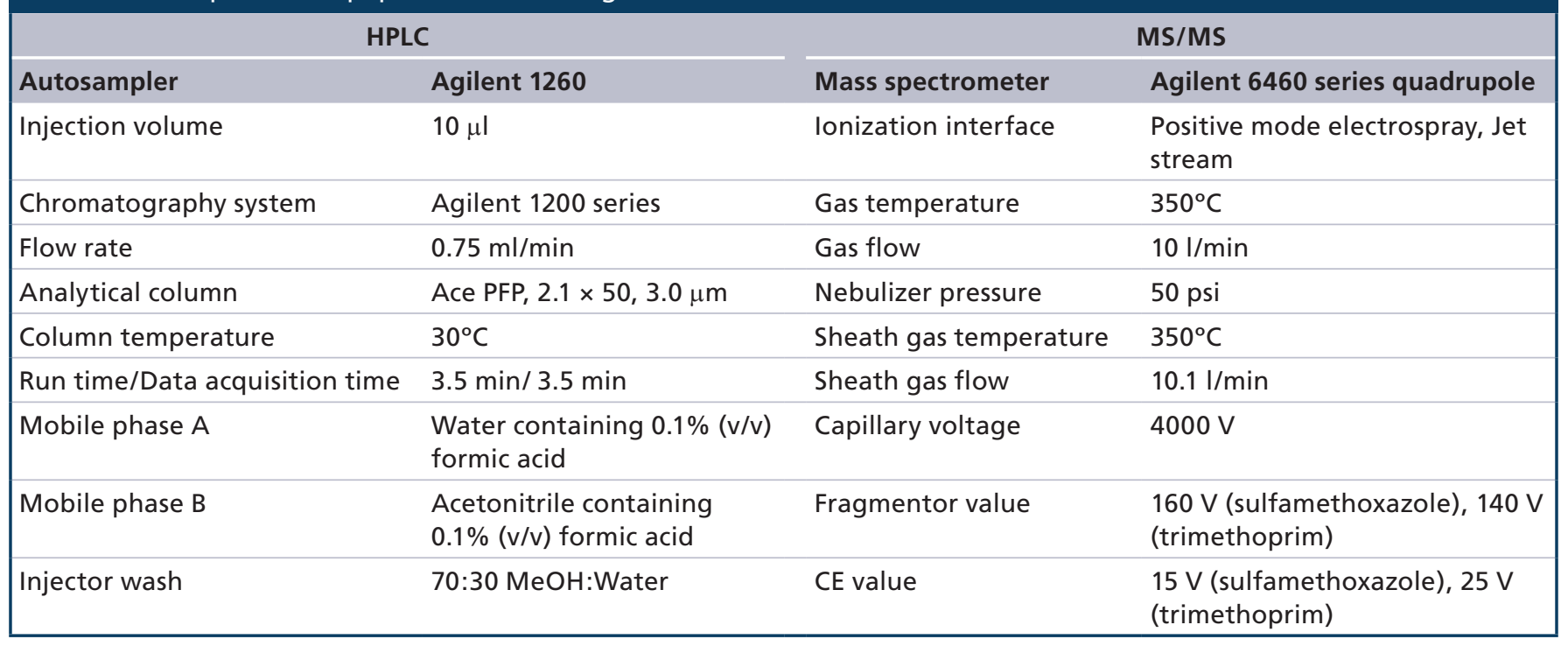


The Agilent 1200 Series HPLC system, Agilent 6410 Series Triple Quadrupole mass spectrometer and Agilent Zorbax XDB-C8 analytical column $(2.1 \mathrm{~mm}$ internal diameter $\times 30 \mathrm{~mm}$ length, $3.5 \mu \mathrm{m}$ particle size) were used (Agilent Technologies). A gradient mobile phase was made up of water containing $0.1 \%$ $(\mathrm{v} / \mathrm{v})$ formic acid and methanol containing $0.1 \%(\mathrm{v} / \mathrm{v})$ formic acid. The method was validated according to the standards set forth by the US FDA [17].

The dried matrix spot and liquid methods were independently developed and validated by two different analysts. The dried matrix spot methods were run on a more sensitive HPLC-MS/MS system because more sensitivity was needed. An explanation for this could be that less volume is in a $6 \mathrm{~mm}$ punch (about 5 $\mu \mathrm{l})$ compared with the $10 \mu \mathrm{l}$ of liquid sample used. The HPLC system is different only because the HPLCs were already configured with the respective mass spectrometers that were used.

\section{Method validation}

The analytical methods were validated according to standards set forth by the FDA [17]. For both DPS and DUS samples, validation included assessment of standard curve fitting, specificity, within- and between-run accuracy and precision, recovery, matrix effect, linearity of dilutions, carryover, punch carryover, sample volume variation, reproducibility and stability. For the latter, bench-top and postpreparative stability results are reported herein.

Specificity was assessed by analysis of samples prepared from human plasma and urine (six different lots each). The method was deemed specific if blank TMP and SMX responses in DPS and DUS samples were $\leq 20 \%$ of the average response at the LLOQ.

Recovery was assessed at three concentrations (low, middle, and high) by comparing extracted QC samples to unextracted QC samples that were prepared by spiking blank matrix postextraction. Matrix effect was evaluated by comparing extracted and spiked solvent QC samples. Carryover was assessed by comparing five replicate injections of the lowest calibration standard followed by five replicate injections of the lower level calibration standard that had each been injected after an ULOQ standard. In addition, punch carryover was assessed by evaluating analyte response with a blank DPS or DUS card that was punched immediately following punching of a card containing the ULOQ. To assess the linearity of dilutions, DPS and DUS samples that were two-times greater than the ULOQ were prepared and diluted ten-times with dried plasma or urine spot extract for analysis. Five replicates were made for each matrix. Sample volume variation was evaluated by spotting mid-concentration QCs using a volume less $(5 \mu \mathrm{l})$ and greater $(15 \mu \mathrm{l})$ than the validated spot volume of $10 \mu \mathrm{l}$. Five replicates were used for each sample volume.

To assess storage stability, low and high QC samples were stored at room temperature in Minigrip zippered bags with desiccant for two or 8 days for DPS samples and two or 14 days for DUS samples. Postpreparative stability for DPS samples was assessed by injecting extracted QC samples (low and high QCs in replicates of five) stored at room temperature for one and 9 days with fresh extracts of calibration standards. Similarly, extracted DUS QC samples (low and high QCs in replicates of five) were stored in the autosampler at room temperature for 4 days and injected with a fresh calibration curve. Samples were deemed stable if the mean values had an accuracy of within $\pm 15 \%$ (i.e., $85-115 \%$ ) and precision did not exceed a coefficient of variation of $15 \%$.

\section{Opportunistic pediatric study}

DPS and DUS clinical samples were collected from pediatric patients enrolled in the Pharmacokinetics of Understudied Drugs Administered to Children per Standard of Care (POPS) trial [18], a multicenter $(n=26)$, prospective, PK and safety study in children ( $<21$ years of age). Children who received one of the targeted drugs of interest (including TMP-SMX) per standard of care as administered by their treating caregiver were eligible for enrollment [19]. Exclusion criteria included failure to obtain consent/assent or known pregnancy. PK samples were collected either at the time of routine clinical laboratory collections or, if the parent/patient consented, at a specific collection time for study purposes. Because this was a standard-of-care study, dosing and PK sample collection times varied between subjects.

Whole blood samples of 200-2000 $\mu$ l were collected based on the participant's age and weight. DPS samples were collected as an aliquot of liquid plasma samples. After plasma was separated via centrifugation ([2000 g] for $10 \mathrm{~min}$ at $4^{\circ} \mathrm{C}$ ), a $10 \mu \mathrm{l}$ micropipette was used to measure 10 microliters per spot, and two plasma spots were spotted per card. The cards were allowed to dry for at least $2 \mathrm{~h}$ at room temperature. The card was then sealed tightly with two desiccant packs and a humidity indicator card in a gas impermeable (Minigrip) bag. The cards were stored and shipped desiccated at ambient temperature. A similar procedure was followed for DUS samples using an aliquot of urine.

\section{Data analysis}

For calibration standards, a plot of the analyte-to-IS peak area ratio versus analyte concentrations was created. A weighted $\left(1 / \mathrm{x}^{2}\right)$ power regression analysis was applied to the data. The concentrations of the analytes 
in the QC samples were determined using the respective calibration line; then, the accuracy and precision of the method was assessed.

An analysis was performed to compare DPS and DUS TMP and SMX concentrations against the established methods of analysis, liquid plasma (LPS) and liquid urine (LUS), respectively. The ratio of DPS to LPS samples (and DUS to LUS) was computed for each drug and plotted against the average concentration for each paired sample. Passing-Bablock regression analysis was used to characterize potential bias between matrices, with dried matrices plotted on the $\mathrm{y}$-axis, and liquid matrices plotted on the $\mathrm{x}$-axis as the reference standard. A Passing-Bablock regression is a nonparametric alternative to ordinary linear regression that assumes a constant ratio of variances, and is less sensitive to outliers when compared with other regression methods [20]. The presence of a systematic and/ or proportional bias was noted if the y-intercept and/ or regression slope significantly differed from zero and unity, respectively [21,22]. Bias also was assessed through calculation of the median percentage prediction error (MPPE), while imprecision was evaluated through calculation of the median absolute percentage prediction error (MAPE). Calculations of MPPE and MAPE were as follows:

$$
\begin{aligned}
& \text { MPPE }=\operatorname{median}\left(100 \% \times\left[\mathrm{C}_{\text {DPS }}-\mathrm{C}_{\text {LPS }}\right] / \mathrm{C}_{\text {LPS }}\right) \\
& \text { MAPE }=\operatorname{median}\left(100 \% \times \mid\left[\mathrm{C}_{\mathrm{DPS}}-\mathrm{C}_{\mathrm{LPS}}\right] / / \mathrm{C}_{\mathrm{LPS}}\right)
\end{aligned}
$$

$\mathrm{C}_{\mathrm{DPS}}$ and $\mathrm{C}_{\mathrm{LPS}}$ denote the drug concentrations in DPS and LPS, respectively. Similar equations were used to calculate MPPE and MAPE for DUS and LUS samples. A value of $<15 \%$ for MPPE or MAPE was considered acceptable [22-24].

STATA 13 (TX, USA) was used for statistical analyses, and MedCalc Statistical Software version 14.8.1 (Ostend, Belgium) was used to generate PassingBablock regression plots. Standard summary statistics were used to describe the demographics and laboratory characteristics of the study population.

\section{Results \& discussion}

\section{Standard curve fitting}

Linear responses (using a power regression) in the TMP/ IS peak area ratios were observed over the range of 100 $50,000 \mathrm{ng} / \mathrm{ml}$ for DPS and 500-250,000 $\mathrm{ng} / \mathrm{ml}$ for DUS samples. Also, linear responses (using a power regression) in the SMX/IS peak area ratios were observed over the range of $1000-500,000 \mathrm{ng} / \mathrm{ml}$ for DPS and DUS samples. The correlation coefficients were 0.995 or better for all runs. Accuracy values were within $\pm 15 \%$ of the theoretical value for all runs at all concentration levels. Representative calibration plots for SMX and TMP in
DPS and DUS are shown in Supplementary Figures $1 \& 2$. Representative chromatograms of LLOQ samples for TMP/SMX in DPS and DUS samples are shown in Supplementary Figure 3. Zero (IS added) and blank (no IS) samples were included with each validation run (Supplementary Figures 4 \& 5).

\section{Specificity}

The characteristic precursor $[\mathrm{M}+\mathrm{H}]^{+}$to product ion transitions, $m / z 291$ to 230 and 294 to 123 for TMP and its IS, and $m / z 254$ to 156 and 258 to 160 for SMX and its IS, were used as multiple reaction monitoring transitions to ensure that optimal selectivity was obtained. For TMP IS, the $294 \rightarrow 123$ transition was used because when we performed the compound optimization, the 123 daughter ion was more abundant and gave us more signal than the 230 daughter ion. The method was deemed to have adequate specificity based on the analysis of TMP and SMX responses in blank samples from six different lots (blank responses $\leq 20 \%$ average response at the LLOQ). No unacceptable interferences at the retention times of TMP, SMX and the IS in DPS or DUS were noted with zero or blank samples. The response observed with LLOQ samples was assessed in three validation runs for both DPS and DUS samples. The following criteria were met for the LLOQ samples: the analyte response was at least five-times the response obtained with blank samples (i.e., $\mathrm{S} / \mathrm{N}$ ratio $\geq 5$ ); peak response had a precision (i.e., CV) of $\leq 20 \%$ and accuracy was between 80 and $120 \%$ of the theoretical value.

\section{Within- \& between-run accuracy \& precision}

Mean within- and between-run accuracy and precision values for TMP-SMX DPS and DUS QC samples are shown in Tables 2 \& 3, respectively. Accuracy was within $\pm 15 \%$ of the theoretical value for all runs. Precision did not exceed $15 \%$ for any run.

\section{Recovery, matrix effect \& linearity of dilutions} Recovery of TMP and SMX in DPS and DUS samples was consistent and reproducible (Table 4). No matrix effect was noted through comparison of the percent recovery observed in extracted QCs with that in spiked solvent samples (100\% recovery). Dilution validation samples also met acceptance criteria based on accuracy and precision.

\section{Carryover \& sample volume variation}

No carryover was noted for TMP and SMX in DPS and DUS samples when the lower level calibration standard was injected following injection of the ULOQ (five replicates each). When punch carryover was assessed, analyte response for blank samples was $>20 \%$ of the mean LLOQ response, and thus the test failed. Punch 
Table 2. Precision and accuracy for dried plasma spots.

\begin{tabular}{|c|c|c|c|c|}
\hline \multirow[t]{2}{*}{ QC sample } & \multicolumn{2}{|c|}{ Trimethoprim } & \multicolumn{2}{|c|}{ Sulfamethoxazole } \\
\hline & Intra-run accuracy (\%) & Intra-run precision (\%) & Intra-run accuracy (\%) & Intra-run precision (\%) \\
\hline \multicolumn{5}{|l|}{ Run 1} \\
\hline Low & 91.6 & 2.8 & 86.4 & 3.9 \\
\hline Mid & 96.6 & 0.8 & 91.6 & 2.0 \\
\hline \multicolumn{5}{|l|}{ Run 2} \\
\hline LLOQ & 93.6 & 3.7 & 89.9 & 5.7 \\
\hline Low & 92.3 & 3.7 & 88.3 & 3.7 \\
\hline Mid & 99.5 & 2.1 & 97.1 & 1.9 \\
\hline Low & 95.4 & 1.7 & 93.3 & 2.1 \\
\hline Mid & 96.4 & 2.5 & 93.0 & 3.7 \\
\hline High & 94.2 & 2.7 & 90.4 & 1.6 \\
\hline QC sample & Inter-run accuracy (\%) & Inter-run precision (\%) & Inter-run accuracy (\%) & Inter-run precision (\%) \\
\hline LLOQ & 93.4 & 2.7 & 89.2 & 5.4 \\
\hline Low & 93.1 & 3.2 & 89.4 & 4.5 \\
\hline Mid & 97.5 & 2.4 & 93.9 & 3.6 \\
\hline High & 95.1 & 2.4 & 90.2 & 3.1 \\
\hline
\end{tabular}

carryover was then reassessed by use of three cleansing punches (using W903 paper) in between punching of the double blank samples. This resulted in a blank response $<20 \%$ of the mean LLOQ response. Spot homogeneity was assessed for each matrix using a 3 $\mathrm{mm}$ punch size. Due to variability in the results, it was determined that a $6 \mathrm{~mm}$ punch must be used to sample the majority of the spot and minimize nonhomogeneity issues. Last, to assess sample volume variation, 5 and 15 $\mu \mathrm{l}$ spot volumes were compared with the validated spot volume (i.e., $10 \mu \mathrm{l}$ ). Accuracy and precision criteria were met for the ten and $15 \mu \mathrm{l}$, but not for the $5 \mu \mathrm{l}(116.3 \%)$.

\section{Stability}

TMP and SMX were stable in DPS when stored at room temperature for 6 days. In DUS, both molecules were stable at room temperature for 12 days. Postpreparative stability results indicated that processed DPS samples are stable for at least 9 days at room temperature. Processed DUS samples were stable for at least 4 days at room temperature. The differences noted between DPS and DUS samples do not reflect actual differences in stability, but instead are a result of the length of time at which the stability was tested.

\section{Comparability analysis}

TMP and SMX concentrations measured in LPS and LUS were compared graphically with observations in DPS and DUS samples, respectively (Figures 2 \& 3).

\section{DPS-to-LPS analysis}

Forty-seven LPS and DPS paired samples were collected from 34 subjects (median [range] age, 6.9 years [0.2, 20.1]; weight, $26.1 \mathrm{~kg}[4.7,139.4]$; TMP dosing, $3.0 \mathrm{mg} / \mathrm{kg} / \mathrm{dose}[0.6,8.8]$ and $3.2 \mathrm{mg} / \mathrm{kg} /$ day [0.6, 17.6]; SMX dosing, $13.8 \mathrm{mg} / \mathrm{kg} /$ dose [3.1, 44.0] and $16.2 \mathrm{mg} / \mathrm{kg} /$ day $[3.1,88.1])$. Four paired TMP samples were below the LOQ.

The mean TMP DPS to LPS ratio was 0.88 (95\% CI: $0.84,0.92$ ), and the limits of agreement were 0.63 and 1.13. Passing-Bablock regression showed linear correlation between the TMP DPS and LPS concentrations, and exhibited negligible 
Table 3. Precision and accuracy for dried urine spots.

Trimethoprim

Intra-run accuracy (\%) Intra-run precision (\%)

Sulfamethoxazole

Intra-run accuracy (\%) Intra-run precision (\%)

Run 1

LLOQ

Low

Mid

High

92.7

101.4

2.5

2.1

2.7

101.5

96.3

4.7

Run 2

LLOQ

Low

Mid

94.0

99.6

101.3

97.4

2.9

1.1

3.6

3.0

9

6

Run 3

LLOQ

99.3

Low

Mid

99.3

102.5

101.7

93.0

Inter-run accuracy (\%)

LLOQ

Low

Mid

High

95.3

100.6

100.9

95.2

Trimethoprim: LLOQ, $500 \mathrm{ng} / \mathrm{ml}$; low, $1500 \mathrm{ng} / \mathrm{ml}$; mid, $20 \mu \mathrm{g} / \mathrm{ml}$; high, $200 \mu \mathrm{g} / \mathrm{ml}$.

Sulfamethoxazole: LLOQ, $1 \mu \mathrm{g} / \mathrm{ml}$; low, $3 \mu \mathrm{g} / \mathrm{ml}$; mid, $40 \mu \mathrm{g} / \mathrm{ml}$; high, $400 \mu \mathrm{g} / \mathrm{ml}$.

Accuracy and precision were assessed using five determinations per concentration. proportional and systematic biases between the two matrices (slope 0.93 [95\% CI: 0.91, 0.97]; intercept -0.04 [95\% CI: $-0.07,-0.02])$. The MPPE for the comparison of TMP DPS to LPS concentrations was $-11.7 \%$ and the MAPE was $12.1 \%$.

The mean SMXDPS to LPS ratio was $0.81(95 \% \mathrm{CI}$ : $0.77,0.86)$, and limits of agreement were 0.51 and
1.12. The Passing-Bablock regression showed modest agreement between the SMX LPS and DPS concentrations (slope 0.86 [95\% CI: 0.80, 0.92]; intercept -0.76 [95\% CI: $-2.02,0.25]$ ), with evidence of proportional bias between the two matrices. MPPE for the comparison of LPS and DPS concentrations was $-19.8 \%$, and the MAPE was $20.3 \%$.

\section{Table 4. Percentage recovery for dried plasma spots and dried urine spots.}

\begin{tabular}{|c|c|c|c|c|}
\hline & \multicolumn{2}{|c|}{ Trimethoprim } & \multicolumn{2}{|c|}{ Sulfamethoxazole } \\
\hline & Dried plasma spots & Dried urine spots & Dried plasma spots & Dried urine spots \\
\hline \multicolumn{5}{|c|}{ Extraction recovery (\%) } \\
\hline Low QC & 103.4 & 100.7 & 100.7 & 97.1 \\
\hline Mid QC & 105.8 & 99.8 & 106.4 & 97.9 \\
\hline High QC & 104.2 & 99.3 & 104.5 & 98.4 \\
\hline \multicolumn{5}{|c|}{$\begin{array}{l}\text { Trimethoprim (DPS): low, } 300 \mathrm{ng} / \mathrm{ml} ; \mathrm{mid}, 4 \mu \mathrm{g} / \mathrm{ml} \text {; high, } 40 \mu \mathrm{g} / \mathrm{ml} \text {. } \\
\text { Trimethoprim (DUS): low, } 1.5 \mu \mathrm{g} / \mathrm{ml} ; \mathrm{mid}, 20 \mu \mathrm{g} / \mathrm{ml} ; \text { high, } 200 \mu \mathrm{g} / \mathrm{ml} \text {. } \\
\text { Sulfamethoxazole (DPS, DUS): low, } 3 \mu \mathrm{g} / \mathrm{ml} ; \mathrm{mid}, 40 \mu \mathrm{g} / \mathrm{ml} ; \text { high, } 400 \mu \mathrm{g} / \mathrm{ml} \text {. } \\
\text { Extraction recovery was assessed using five determinations per concentration. } \\
\text { DPS: Dried plasma spots; DUS: Dried plasma spots. }\end{array}$} \\
\hline
\end{tabular}




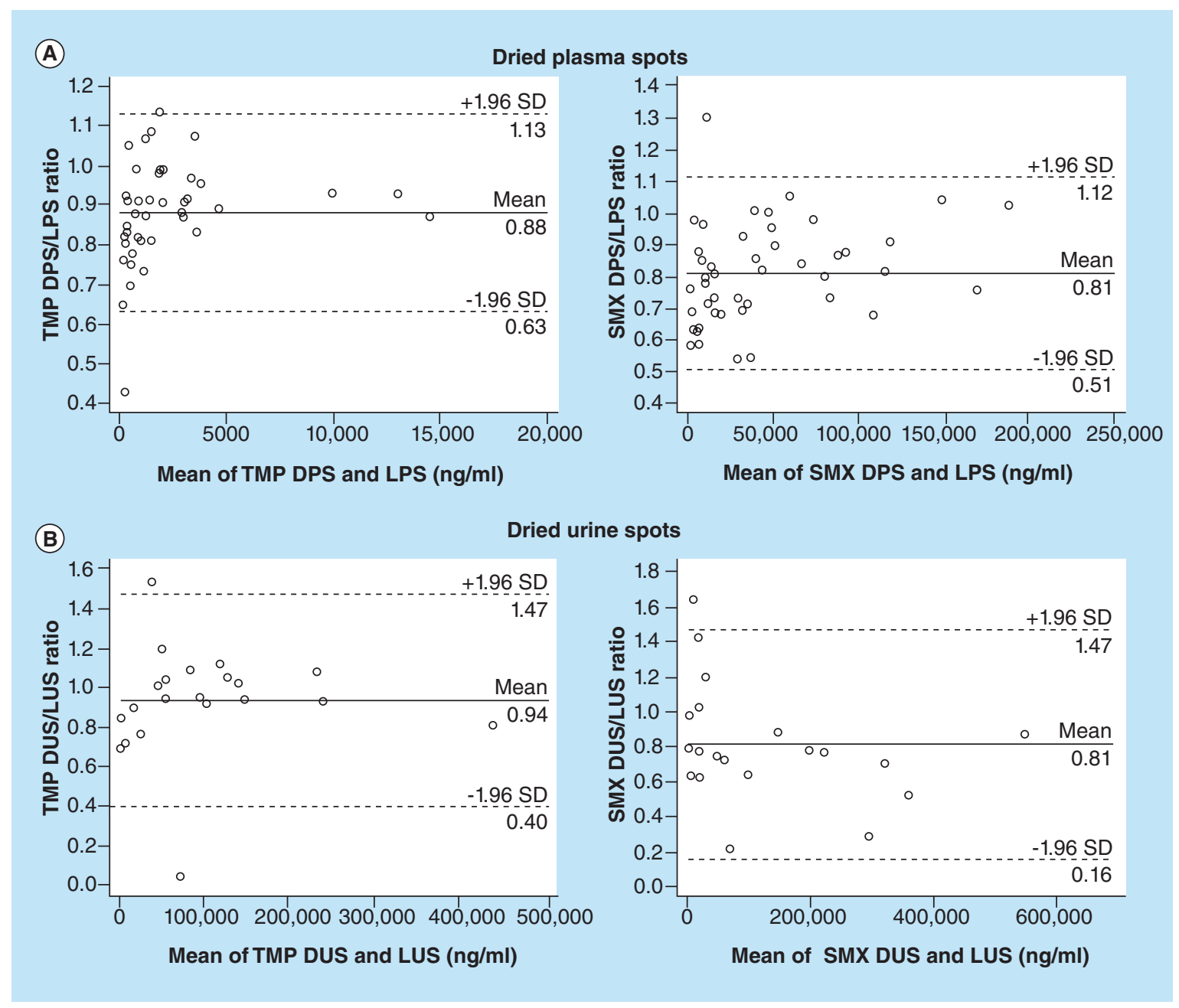

Figure 2. Plot of the dried matrix sample to liquid sample concentration ratio versus the mean of the paired concentration. (A) Plot of dried plasma spot-to-liquid plasma sample ratios versus the mean of the dried plasma spot and liquid plasma sample concentrations. (B) Plot of dried urine spot-to-liquid urine sample ratios versus the mean of the dried urine spot and liquid urine sample concentrations. The dark solid line represents the observed mean ratio, and the dashed lines reference the limits of agreement between the samples.

DPS: Dried plasma spot; DUS: Dried urine spot; LPS: Liquid plasma sample; LUS: Liquid urine sample;

SMX: Sulfamethoxazole; TMP: Trimethoprim.

\section{DUS-to-LUS analysis}

Twenty-one LUS and DUS paired samples were collected from 16 subjects (median [range] age, 13.9 years [2.3, 20.1]; weight, $43.2 \mathrm{~kg}$ [12.8, 86.0]; TMP dosing, $3.4 \mathrm{mg} / \mathrm{kg} /$ dose $[0.6,8.8]$ and $6.1 \mathrm{mg} / \mathrm{kg} /$ day $[0.6$, 17.6]; SMX dosing, $17.0 \mathrm{mg} / \mathrm{kg} / \mathrm{dose}[3.1,44.0]$ and $30.5 \mathrm{mg} / \mathrm{kg} / \mathrm{day}[3.1,88.1])$. One paired SMX sample was below the limit of quantification.

The mean TMP DUS to LUS ratio was 0.94 (95\% CI: $0.81,1.06$ ), and the limits of agreement were 0.40 and 1.47. Passing-Bablock regression showed linear correlation between the TMP DUS and LUS concentrations and exhibited no evidence of proportional or systematic bias between the two matrices (slope 0.96 [95\% CI: $0.90,1.08]$; intercept -0.26 [95\% CI: -2.78 , 5.21]). The MPPE for the comparison of TMP DUS to LUS concentrations was $-6.42 \%$ and the MAPE was $17.52 \%$.

The mean SMX DUS to LUS ratio was 0.81 (95\% CI: $0.66,0.97)$ and limits of agreement were 0.16 and 1.47. The Passing-Bablock regression showed modest agreement between the SMX LUS and DUS concentrations (slope 0.71 [95\% CI: 0.58, 0.79]; intercept 1.16 [95\% CI: $-1.66,8.56]$ ), with evidence of proportional bias between the two matrices. MPPE for the comparison of SMX LUS and DUS concentrations was $-18.66 \%$ and the MAPE was $31.47 \%$.

\section{Conclusion}

An accurate, precise and selective method was developed to detect TMP and SMX in DPS and DUS samples. From a clinical trial perspective, the devel- 


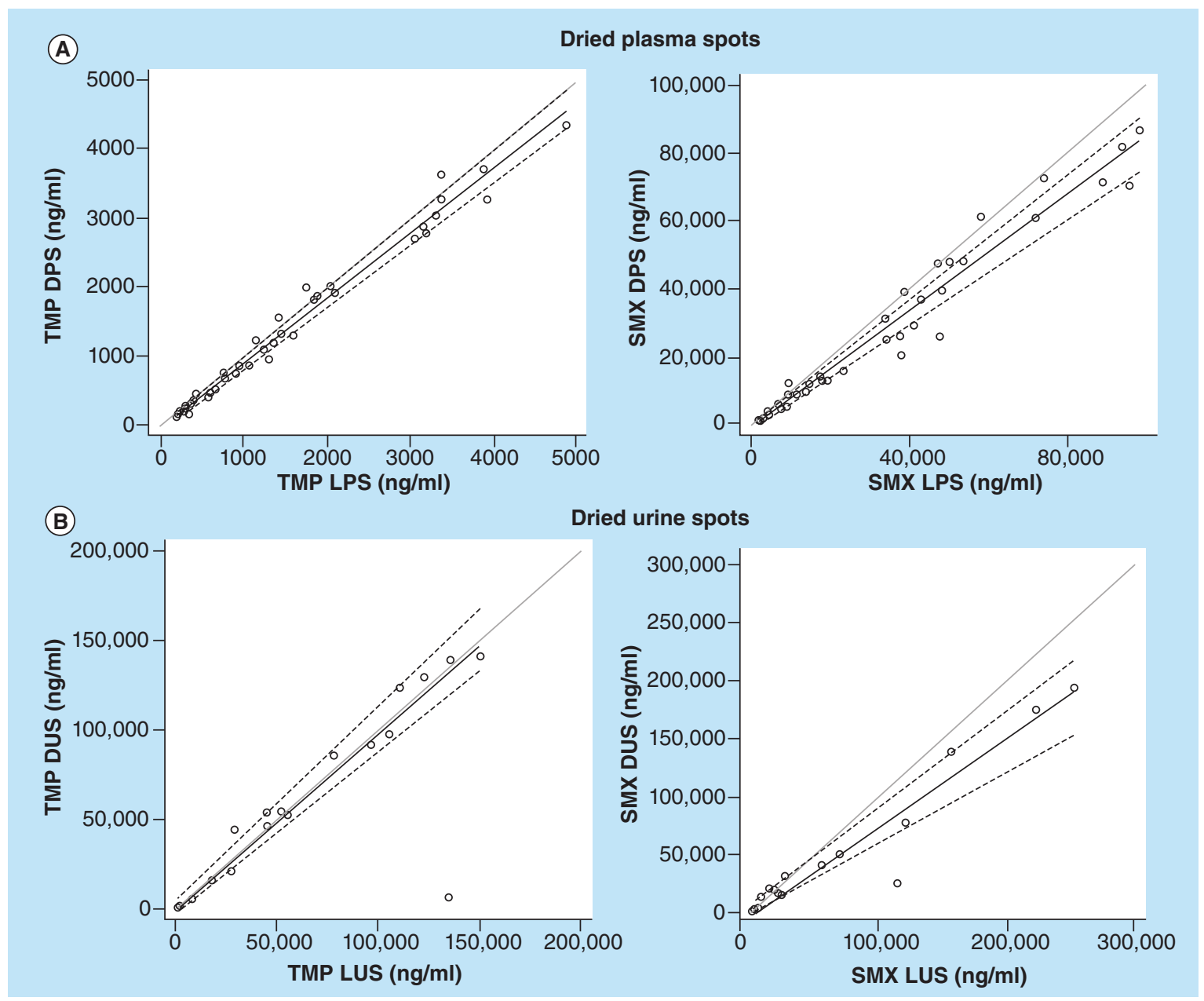

Figure 3. Passing-Bablock regression of trimethoprim and sulfamethoxazole dried plasma and urine spots versus liquid plasma and urine sample concentrations (gray line represents line of unity; black line is the regression line; dashed lines represent $95 \% \mathrm{CI}$ ). (A) Liquid plasma sample versus dried plasma spot concentrations for trimethoprim and sulfamethoxazole. (B) Liquid urine sample versus dried urine spot concentrations for trimethoprim and sulfamethoxazole.

DPS: Dried plasma spot; DUS: Dried urine spot; LPS: Liquid plasma sample; LUS: Liquid urine sample; SMX: Sulfamethoxazole; TMP: Trimethoprim.

oped dried matrix sampling method has advantages over traditional liquid methods because it allows for more flexible sample collection and storage, as well as reduced sample volumes. In the assay validation process, it was determined that three cleansing punches and a $6 \mathrm{~mm}$ punch size were optimal to prevent punch carryover and nonhomogeneity issues. The developed method was applied successfully to measure TMPSMX in PK samples collected from an opportunistic PK study. The LLOQ of the assay was appropriate $(100 \mathrm{ng} / \mathrm{ml}$, DPS TMP; $500 \mathrm{ng} / \mathrm{ml}$, DUS TMP; $1000 \mathrm{ng} / \mathrm{ml}$, DPS/DUS SMX).

LPS and DPS yield similar concentrations of TMP as evidenced by a linear relationship on the scatter plot. While similar, however, TMP LPS and DPS concentrations were not identical as evidenced by a negligible deviation in the mean DPS/LPS ratio (0.88) and slope of the Passing-Bablock regression (0.93). Interpretation of the Passing-Bablock regression indicates that the differences between the two methods are both systematic and proportional, such that DPS concentrations are systematically lower than LPS values throughout the concentration range, but this difference is more marked at higher concentrations. When a correction factor of 0.88 (mean ratio of DPS/ LPS) is applied to the DPS concentrations, the fit of the Passing-Bablock regression is improved slightly (slope 1.06, 95\% CI: 1.03, 1.1; intercept -0.04, 95\% CI: -0.08, -0.02). For TMP, DUS and LUS concentrations of TMP were nearly interchangeable. Based on these results, dried matrix sampling is an appropriate method to assess TMP PK in clinical studies.

For SMX, LPS and DPS matrices yield modest agreement as evidenced by a relatively linear relationship 
on the scatter plot. However, SMX LPS and DPS concentrations deviate slightly from each other as evidenced by the mean DPS/LPS ratio and slope of the Passing-Bablock regression (0.86). Interpretation of the Passing-Bablock regression indicates the presence of proportional bias such that the relationship between DPS and LPS concentrations are more variable at higher concentrations. Application of a correction factor of 0.81 (DPS concentration was divided by the mean ratio of DPS/LPS) improved the fit of the Passing-Bablock regression, and proportional bias is no longer apparent (slope 1.07, 95\% CI: 0.98, 1.14; intercept $0.94,95 \% \mathrm{CI}:-2.49,0.30$ ). A similar proportional bias was observed with SMX DUS samples, and application of a correction factor of 0.81 (mean ratio of DUS/LUS) improved the fit of the PassingBablock regression and decreased proportional bias, though this bias remains apparent (slope $0.88,95 \%$ CI: 0.71, 0.98; intercept 1.43, 95\% CI: $-2.05,10.57$ ). However, due to the bias and imprecision observed between matrices, further study is needed before dried matrix sampling can be applied to assess SMX PK in clinical studies.

The present findings may be explained by a number of considerations. The physicochemical properties of TMP and SMX, and interaction with the filter paper, may contribute to differences in drug recovery from dried matrix versus liquid samples. The possibilities of inadequate recovery have been demonstrated with the drug, gabapentin. Its zwitterionic properties require special processes of drug extraction from the dried matrix as well as cleaning thereafter [25]. Previous investigations of everolimus concentrations in DBS also have demonstrated a concentration-dependent level of drug extraction from the dried matrix [26]. In this study, in the clinical samples analyzed, extraction of drug at low concentrations was higher compared with extraction of drug at higher concentrations. This finding is similar to the proportionality observed in our evaluation of TMP and SMX concentrations, although in the method validation there was no evidence of concentration-dependent extraction. Proportionality has been improved previously by changes in sample preparation, including prolonged shaking time and ultrasonic vibration, as well as modifying methods of sample extraction [27]. Alternatively, we were able to demonstrate improvement in proportionality with the addition of a mathematical correction factor.

A drug's degree of protein binding may mediate some interactions with the filter paper and the ability to extract drug from the dried matrix. The differential protein binding between TMP (40\%) and SMX $(70 \%)$ could potentially explain some of the increased variability observed in SMX compared with TMP evaluations [28]. Previous investigators have demonstrated that the addition of pasteurized plasma proteins to the filter paper used in DBS has been useful to deactivate the sites of absorption on the filter paper, making it easier to extract drug [29]. Chemicals also have been used to denature proteins and help promote drug extraction [30]. The benefit of these processes is the ability to potentially increase drug recovery; however, the addition of pasteurized proteins or other chemicals to the dried matrix also may contribute to matrix effects and interfere with the accuracy of the correlation of drug concentrations between liquid and dried plasma matrices.

The method of assessment of comparability has been a point of contention and discussion in the literature [22,31]. Previous authors have stressed the importance of consideration of linearity, normal versus nonparametric distribution of the data, and the potential for misclassification of method bias with the wrong method of comparability [22,31]. The methods used in this analysis are thought to be beneficial because of the lack of sensitivity to outliers and nonparametric methodology. In addition, the Passing-Bablock method has been used in previous comparisons of dried and liquid matrices, with good and consistent results [22,32]. Use of multiple methods in this analysis revealed similar findings for each drug, thereby confirming the appropriateness of the methodology.

\section{Future perspective}

Pediatric PK trials are challenging because of the limitations surrounding sparse sampling and blood volume requirements. Dried matrix sampling is an innovative approach to collect PK samples using limited blood volume. To be successful, dried matrix samples must be coupled with a highly sensitive analytical method (due to small sample volumes). When available, dried matrix PK samples can be collected to characterize drug disposition in children while minimizing the ethical concerns related to blood sampling in this very vulnerable population. Also, because of the ease of sample collection and processing with this innovative technology, samples can be collected and stored more effectively relative to wet matrices. For drugs where concentrations in dried matrix samples can serve as a surrogate for plasma concentrations, collection of DPS/DUS samples alone can be used to characterize the PK of drugs in children. DPS samples have the advantage of avoiding the 'hematocrit effect' on blood spot homogeneity, matrix effects and assay variability, which can be observed with DBS sample analysis. 


\section{Supplementary data}

To view the supplementary data that accompany this paper please visit the journal website at: www.future-science.com/ doi/full/10.4155/BIO.15.38

\section{Disclaimer}

The content is solely the responsibility of the authors and does not necessarily represent the official views of the $\mathrm{NIH}$.

\section{Acknowledgements}

The Pediatric Trials Network Administrative Core Committee: K Y Berezny, Duke Clinical Research Institute, Durham, NC, USA; E Capparelli, University of California-San Diego, San Diego, CA, USA; G L Kearns, Children's Mercy Hospital, Kansas City, MO, USA; M Laughon, University of North Carolina at Chapel Hill, Chapel Hill, NC, USA; A Muelenaer, Virginia Tech Carilion School of Medicine, Roanoke, VA, USA; T M O'Shea, Wake Forest Baptist Medical Center, Winston Salem, NC, USA; I M Paul, Penn State College of Medicine, Hershey, PA, USA; P B Smith, Duke Clinical Research Institute Durham, NC, USA; J van den Anker, George Washington University School of Medicine and Health, Washington, DC, USA; K Wade, Children's Hospital of Philadelphia, Philadelphia, PA, USA; T J Walsh, Weill Cornell Medical College of Cornell University, New York, NY, USA. The Eunice Kennedy Shriver National Institute of Child Health and Human Development: D Siegel, P Taylor-Zapata, A Zajicek, Z Ren, K Tsilou, A Pagan. The EMMES Corporation (Data Coordinating (enter): R Anand, G Simone. Pediatric Trials Network study team, principal investigators \& study coordinators: A Lewandowski, EMMES Corporation, Rockville, MD, USA; B Harper, Duke Clinical Research Institute, Durham, NC, USA; $B$ Poindexter and L Smiley, Riley Hospital for Children, Indianapolis, IN,USA; R Yogev and L Fern, Ann \& Robert H. Lurie Children's Hospital, Chicago, IL, USA; Andrew Atz and H Al Nasiri, Medical University of South Carolina Children's Hospital, Charleston, SC, USA; J Sullivan and T Bratton, University of Louisville and Kosair Children's Hospital, Louisville, KY, USA; Susan Mendley and Donna Cannonier, University of Maryland Hospital, Baltimore, MD, USA; Paula Delmore, Wesley Medical Center, Wichita, KS, USA.

\section{Financial \& competing interest disclosure}

This work was funded under National Institute of Child Health and Human Development (NICHD) contract $\mathrm{HH}$ SN201000003I for the Pediatric Trials Network (PI: Benjamin) and HHSN27500006 (PI: Melloni, Cohen-Wolkowiez) for the Pharmacokinetics of Understudied Drugs Administered to Children per Standard of Care Study (POPS; protocol NICHD-2011-POP01). Research reported in this publication also was supported by the National Center for Advancing Translational Sciences of the National Institutes of Health (NIH) under award number UL1TR001117. The assay measuring TMP-SMX concentrations was performed at OpAns Laboratory (Durham, NC, USA). D Gonzalez is funded by training grant T32GM086330 from the National Institute of General Medical Sciences (NIGMS). D K Benjamin Jr receives support from the US government for his work in pediatric and neonatal clinical pharmacology (2K24HD058735-06, UL1TR001117, NICHD contract HHSN275201000003I and NIAID contract HHSN2722015000061); he also receives research support from Cempra Pharmaceuticals (subaward to HHSO100201300009C) for neonatal and pediatric drug development (www.dcri.duke.edu/research/coi.jsp). M Cohen-Wolkowiez receives support for research from the NIH (1R01-HD076676-01A1), the National Center for Advancing Translational Sciences of the NIH (UL1TR001117), the National Institute of Allergy and Infectious Disease (HHSN272201500006I and HHSN272201300017I), the National Institute for Child Health and Human Development of the NIH (HHSN275201000003I), the FDA (1U01FD004858-01), the Biomedical Advanced Research and Development Authority (HHSO100201300009C), the nonprofit organization Thrasher Research Fund (www.thrasherresearch.org) and from industry (CardioDx and Durata Therapeutics) for drug development in adults and children (www.dcri.duke.edu/research/coi.jsp). The remaining authors have no funding to disclose. The authors have no other relevant affiliations or

\section{Executive summary}

- A precise, accurate and reproducible HPLC-MS/MS method was validated and applied to simultaneously quantify trimethoprim-sulfamethoxazole in dried plasma spots and dried urine spots samples collected in an opportunistic pediatric study.

- The LLOQ for trimethoprim was $100 \mathrm{ng} / \mathrm{ml}$ in dried plasma spots and $500 \mathrm{ng} / \mathrm{ml}$ in dried urine spots samples; for sulfamethoxazole, the LLOQ was $1000 \mathrm{ng} / \mathrm{ml}$ for both matrices.

- Three cleansing punches and a $6 \mathrm{~mm}$ punch size were optimal to prevent punch carryover and homogeneity issues, respectively.

- Dried matrix sampling is an accurate and precise option for evaluation of trimethoprim concentrations in pediatric patients.

- Optimal bioanalysis conditions must be assessed and the application of this approach must be evaluated in clinical trials before there is widespread implementation of dried matrix sampling for drug measurement in children. 
financial involvement with any organization or entity with a financial interest in or financial conflict with the subject matter or materials discussed in the manuscript apart from those disclosed.

The authors thank A McMillan for her editorial assistance. A McMillan was funded by HHSN27500006 (PI: Melloni, Cohen-Wolkowiez).

\section{References}

Papers of special note have been highlighted as either:

- of interest $\bullet$ of considerable interest.

1 Libecco JA, Powell KR. Trimethoprim/sulfamethoxazole: clinical update. Pediatr. Rev. 25(11), 375-380 (2004).

2 Herigon JC, Hersh AL, Gerber JS, Zaoutis TE, Newland JG. Antibiotic management of Staphylococcus aureus infections in US children's hospitals, 1999-2008. Pediatrics 125(6), E1294-E1300 (2010).

3 Sayar E, Sahin S, Cevheroglu S, Atilla Hincal A. Development and validation of an HPLC method for simultaneous determination of trimethoprim and sulfamethoxazole in human plasma. Eur. J. Drug Metab. Pharmacokinet. 35(1-2), 41-46 (2010).

4 Essers L, Korte H. Comparison of the conventional methods and high-performance liquid chromatography for the determination of trimethoprim, sulfamethoxazole and its metabolite in. Chemotherapy 28(4), 247-252 (1982).

5 DeAngelis D, Woolley J, Sigel C. High-performance liquid chromatographic assay for the simultaneous measurement of trimethoprim and sulfamethoxazole in plasma or urine. Ther. Drug Monit. 12(4), 382-392 (1990).

6 Amini H, Ahmadiani A. Rapid and simultaneous determination of sulfamethoxazole and trimethoprim in human plasma by high-performance liquid chromatography. J. Pharm. Biomed. Anal. 43(3), 11461150 (2007).

7 Bedor D, Gonçalves T, Ferreira M et al. Simultaneous determination of sulfamethoxazole and trimethoprim in biological fluids for high-throughput analysis: comparison of HPLC with ultraviolet and tandem mass spectrometric detection. J. Chromatogr. B. 863(1), 46-54 (2008).

8 Stein GE, Throckmorton JK, Scharmen AE et al. Tissue penetration and antimicrobial activity of standard- and high-dose trimethoprim/sulfamethoxazole and linezolid in patients with diabetic foot infection. J. Antimicrob. Chemother. 68(12), 2852-2858 (2013).

9 Teshima D, Otsubo K, Makino K, Itoh Y, Oishi R. Simultaneous determination of sulfamethoxazole and trimethoprim in human plasma by capillary zone electrophoresis. Biomed. Chromatogr. 18(1), 51-54 (2004).

10 Laughon MM, Benjamin DK, Capparelli E V et al. Innovative clinical trial design for pediatric therapeutics. Expert Rev. Clin. Pharmacol. 4(5), 643-652 (2011).

- This article discusses the challenges of performing pediatric clinical trials and the potential value of using dried matrix sampling in this context.
Ethical conduct of research

The authors state that they have obtained appropriate institutional review board approval or have followed the principles outlined in the Declaration of Helsinki for all human or animal experimental investigations. In addition, for investigations involving human subjects, informed consent has been obtained from the participants involved.

11 Meesters R, Hooff G. State-of-the-art dried blood spot analysis: an overview of recent advances and future trends. Bioanalysis 5(17), 2187-2208 (2013).

- This article highlights technological advancements in dried blood spot bioanalysis.

12 Kostić N, Dotsikas Y, Jovi N, Stevanovi G, Malenovi A, Medenica M. Vigabatrin in dried plasma spots: validation of a novel LC-MS/MS method and application to clinical practice. J. Chromatogr. B. Analyt. Technol. Biomed. Life Sci. 962, 102-108 (2014).

13 Ikeda K, Ikawa K, Yokoshige S, Yoshikawa S, Morikawa N. Gas chromatography-electron ionization-mass spectrometry quantitation of valproic acid and gabapentin, using dried plasma spots, for therapeutic drug monitoring in in-home medical care. Biomed. Chromatogr. 28(12), 1756-1762 (2014).

14 Baietto L, D’Avolio A, Pace S et al. Development and validation of an UPLC-PDA method to quantify daptomycin in human plasma and in dried plasma spots. J. Pharm. Biomed. Anal. 88, 66-70 (2014).

www.ncbi.nlm.nih.gov/pubmed/24036034

15 Ramesh T, Nageswara Rao P, Nageswara Rao R. Development of LC-MS/MS method for the determination of dapiprazole on dried blood spots and urine: application to pharmacokinetics. Biomed. Chromatogr. 28(5), 615-620 (2014).

16 De Vries R, Barfield M, van de Merbel N et al. The effect of hematocrit on bioanalysis of DBS: results from the EBF DBS-microsampling consortium. Bioanalysis 5(17), 2147-2160 (2013).

17 FDA. Guidance for industry: bioanalytical method validation. CDER Rockville, MD, USA (2001). www.fda.gov

18 ClinicalTrials Database: NCT01431326. https://clinicaltrials.gov/ct2/show/NCT01431326

19 Gonzalez D, Melloni C, Yogev R et al. Use of opportunistic clinical data and a population pharmacokinetic model to support dosing of clindamycin for premature infants to adolescents. Clin. Pharmacol. Ther. 96(4), 429-437 (2014).

20 Stöckl D, Dewitte K, Thienpont LM. Validity of linear regression in method comparison studies: is it limited by the statistical model or the quality of the analytical input data? Clin. Chem. 44(11), 2340-2346 (1998).

21 Passing H, Bablok W. Method comparison studies and determination of sample sizes application of linear regression procedures for method comparison studies in clinical chemistry. Part. Clin. Chem. Lab. Med. 22(6), 2014 (1984).

22 Parsons TL, Marzinke MA, Hoang T et al. Quantification of rifapentine, a potent anti-tuberculosis drug, from dried blood spot samples using liquid chromatographic-tandem mass 
spectrometric analysis. Antimicrob. Agents Chemother. 58(11), 6747-6757 (2014).

- This article describes development of an LC-MS/MS method for quantification of rifapentine in dried blood spots and an assessment of comparability between paired dried blood spot and liquid plasma samples.

23 David OJ, Johnston A. Limited sampling strategies for estimating cyclosporin area under the concentration-time curve: review of current algorithms. Ther. Drug Monit. 23(2), 100-114 (2001).

24 Ting LSL, Villeneuve E, Ensom MHH. Beyond cyclosporine: a systematic review of limited sampling strategies for other immunosuppressants. Ther. Drug Monit. 28(3), 419-430 (2006).

25 Kolocouri F, Dotsikas Y, Loukas YL. Dried plasma spots as an alternative sample collection technique for the quantitative LC-MS/MS determination of gabapentin. Anal. Bioanal. Chem. 398(3), 1339-1347 (2010).

26 Van der Heijden J, de Beer Y, Hoogtanders K et al. Therapeutic drug monitoring of everolimus using the dried blood spot method in combination with liquid chromatography-mass spectrometry. J. Pharm. Biomed. Anal. 50(4), 664-670 (2009).
27 Allanson A, Cotton MM, Tettey JNA, Boyter A. Determination of rifampicin in human plasma and blood spots by high performance liquid chromatography with UV detection: a potential method for therapeutic drug monitoring. J. Pharm. Biomed. Anal. 44(4), 963-969 (2007).

28 Bactrim DS product label. http://dailymed.nlm.nih.gov

29 Li W, Tse FL. Dried blood spot sampling in combination with LC-MS/MS for quantitative analysis of small molecules. Biomed. Chromatogr. 24(1), 49-65 (2010).

30 Luckwell J, Danielsson A, Johnson B, Clegg S, Green M, Pierce A. Applications and chemistry of cellulose papers for dried blood spots. In: Dried Blood Spots: Applications and Techniques (1st Edition). Wiley, NJ, USA (2014).

31 Bland JM, Altman D. Statistical methods for assessing agreement between two methods of clinical measurement. Lancet 1(8476), 307-310 (1986).

32 Baietto L, D’Avolio A, Ariaudo A et al. Development and validation of a new UPLC-PDA method to quantify linezolid in plasma and in dried plasma spots. J. Chromatogr. B. Analyt. Technol. Biomed. Life Sci. 936, 42-47 (2013).

\section{Affiliations}

\section{- Daniel Gonzalez}

Division of Pharmacotherapy \& Experimental Therapeutics, UNC Eshelman School of Pharmacy, University of North Carolina, Chapel Hill, NC, USA

- Chiara Melloni

Duke Clinical Research Institute, Duke University Medical Center, Durham, NC, USA

- Brenda B Poindexter

Department of Pediatrics, Riley Hospital for Children, Indiana University, Indianapolis, IN, USA

- Ram Yogev

Ann \& Robert H Lurie Children's Hospital of Chicago, Feinberg School of Medicine, Northwestern University, Chicago, IL, USA

- Andrew M Atz

Department of Pediatrics, Medical University of South Carolina, Charleston, SC, USA

- Janice E Sullivan

Kosair Charities Pediatric Clinical Research Unit, Department of Pediatrics, University of Louisville, Kosair Children's Hospital, Louisville, KY, USA

- Susan R Mendley

Department of Pediatrics, University of Maryland School of Medicine, Baltimore, MD, USA

- Paula Delmore

Department of Neonatology, Wesley Medical Center, Wichita, KS, USA

- Amy Delinsky

OpAns, LLC, 4134 S Alston Ave \# 101, Durham, NC 27713, USA

- Kanecia Zimmerman

Duke Clinical Research Institute, Duke University Medical Center, Durham, NC, USA

- Andrew Lewandowski

EMMES Corp., 401 N Washington St \# 700, Rockville, MD 20850, USA

- Barrie Harper

Duke Clinical Research Institute, Duke University Medical Center, Durham, NC, USA

- Kenneth C Lewis

OpAns, LLC, 4134 S Alston Ave \# 101, Durham, NC 27713, USA

- Daniel K Benjamin Jr

Duke Clinical Research Institute, Duke University Medical Center, Durham, NC, USA and

Department of Pediatrics, Duke University Medical Center, Durham, NC, USA

- Michael Cohen-Wolkowiez

Duke Clinical Research Institute, Duke University Medical Center, Durham, NC, USA

and

Department of Pediatrics, Duke University Medical Center, Durham, NC, USA 